\title{
Shape Control in Gold Nanoparticles by N-Containing Ligands: Insights from Density Functional Theory and Wulff Constructions
}

\author{
M. Domingo ${ }^{1} \cdot$ M. Shahrokhi ${ }^{1} \cdot$ I. N. Remediakis ${ }^{2,3} \cdot$ N. Lopez $^{1}$
}

\begin{abstract}
The controlled growth promoted by the use of ligands can affect the structural properties of nanoparticles, preferential growth and most likely exposed facets in their final shape. The chemistry is deeply dominated by the close relationship between both the interaction of the ligands and the metal structure. In the present work, we have illustrated the change in the nanoparticle shape as a function of a series of nitrogen bases. Particularly, we have employed Density Functional Theory to obtain the interaction energies of a series of nitrogen containing bases to gold surfaces with different orientations. The adsorption strength is found to correlate with the HOMO position of the ligand thus providing a fast screening tool for this property. Moreover, for small N-bases with high $\mathrm{N}$ content we have found that the shape can be tuned as a function of the coverage and the final structure at high coverages severely departs from that of bare gold nanoparticles. We have found variations in the different extension of the facets that can be further employed in obtaining structure sensitivity and the right chemical and catalytic performance.
\end{abstract}

Keywords Gold nanoparticles · N-containing ligands · Density Functional Theory (DFT) · Wulff construction

\section{Introduction}

Gold chemistry has intrigued scientists in both homogeneous and heterogeneous catalysis for more than two decades now [1-4]. Particularly, when prepared highly porous, the catalytic activity is more prominent likely due to the presence of a larger fraction of low coordinated sites [5-8]. Most of the mechanisms for the reactivity on clean metals or organometallic compounds have been clarified [9] but there is still a wide open area regarding ligand coated nanoparticles that might abridge the properties between the two materials.

Among the most likely properties, ligand-induced shape control is the most obvious. Colloidal chemistry has a rich toolbox that allows the synthesis of different

N. Lopez

nlopez@iciq.es

1 Institute of Chemical Research of Catalonia, ICIQ, The Barcelona Institute of Science and Technology, Av. Països Catalans 16, 43007 Tarragona, Spain

2 Department of Materials Science and Technology, University of Crete, Heraklion, Greece

3 Institute of Electronic Structure and Laser, FORTH, Heraklion, Greece nanoarchitectures by changing the nature of the ligands. However, the intimate mechanisms that apply are not known in a large detail in part because of the multiscale nature of the problem of nucleation and growth [10]. A very successful approach however is the use of Density Functional Theory, DFT, to understand the very fundamental modifications induced at the atomistic scale by the adsorption of ligands. Indeed, employing traditional methodologies based on a continuum model that allows the calculation of the surface energy via DFT and its combination with ab-initio thermodynamics has proven a useful tool when assessing the tridimensional structure of nanoparticles [11-14] for a large variety of systems and including concave nanoparticles [15]. As reactions are often differently promoted by facet orientation (structure sensitivity) the shape control of the nanocatalyst is of primarily interest.

Among all ligands $\mathrm{N}$-containing ones have emerged as the most suitable for further co-catalytic activity [16]. The reason behind the activity of these $\mathrm{N}$-bases as ligands can be traced back to the fact that when adsorbed on a metal surface they form frustrated Lewis pairs (FLP) $[17,18]$ that allow the easy activation of $\mathrm{H}_{2}$ otherwise impossible for bulk $\mathrm{Au}$. This concept has been recently reviewed [19]. Therefore, the study of the interaction of a series of N-bases on different 
facets of gold is mandatory to identify if the presence of these ligands affects also the overall shape of the gold nanoparticles thus adding to the electronic contribution of the FLP a modulation different facets in the metal nanoparticles.

In the present work, we have taken a list of the most common N-bases with different hapticity to evaluate the effects of the ligands on the exhibited surfaces. The molecules are: aniline, ethylenediamine, pyrazine, pyridine, pyrrole, trimethylamine and porphine, see Scheme 1. By means of Density Functional Theory we have computed the adsorption of these ligands to the low-Miller-index facets of gold. The ligand-decorated surface energies have then been employed in the Wulff and atomistic Wulff construction to derive the equilibrium shape of the corresponding gold nanoparticles.

\section{Computational Details}

We have performed Density Functional Theory calculations using the Vienna ab initio simulation package code (VASP) [20, 21] with the Perdew-Burke-Enzerhof (PBE) exchange-correlation functional [22]. The interaction between the valence and the core electrons was described with the projected augmented wave (PAW) method with the implementation of Kresse and Joubert [23]. The energy cutoff was set to $450 \mathrm{eV}$ and van der Waals contributions were described using the semi-empirical DFT-D2 approach [24, 25] and the parameters were reoptimized for this metal in our group [26]. The calculations of surface energy were carried out for various terminations of three low-index planes: (001), (110) and (111) using periodic boundary conditions. The models for (100) and (111) Au surface were constructed with five atomic layers while (110) was modeled with 9 atomic layers where the two topmost layers were fully relaxed and the others were fixed to their bulk positions. To ensure the decoupling of the adjacent slabs, a $12 \AA$ thick vacuum region was employed for all slabs. The N-ligands were adsorbed on the topmost layer and dipole correction was used. For all ligand molecules adsorption except porphine, the (100), (110) and (111) Au surfaces were modeled by a slab consisting of a $4 \times 4,3 \times 3$, and $4 \times 4$ surface unit cell, respectively, and were optimized using a $(3 \times 3 \times 1),(2 \times 3 \times 1)$, and $(3 \times 3 \times 1) k$-point mesh. For porphine adsorption, a slab model consisting $5 \times 5,4 \times 5$ and $5 \times 5$ surface unit cell were used for the (100), (110) and (111) facets and optimized using a $(3 \times 3 \times 1),(2 \times 3 \times 1)$, and $(3 \times 3 \times 1)$ $k$-point mesh, respectively. All the computed structures have been incorporated to the ioChem-BD database [27] and can be retrieved through the following https://doi.org/10.19061/ iochem-bd-1-49.

\section{Results}

In this paper, we discuss the characteristics of each Au surface and the adsorption behavior of different ligands on all of them. We begin by calculating the surface energy, $\gamma_{s}$, for $\mathrm{Au}$ by simulations of periodic slabs. For a given model slab of a facet featuring Miller index $(h k l)$, the $\gamma_{s}$ is given by the following expression:

$\gamma_{s}=\frac{1}{2 A}\left[E_{\text {slab }}^{\text {unrelax }}-N E_{\text {bulk }}\right]+\frac{1}{A}\left[E_{\text {slab }}^{\text {relax }}-E_{\text {slab }}^{\text {unrelax }}\right]$

where $A$ is area of the surface considered, $E_{\text {slab }}^{\text {unrelax }}$ and $E_{\text {slab }}^{\text {relax }}$ are the respective total energies of the unrelaxed and relaxed $N$-atom slabs, $N$ is the number of atoms in the slab,

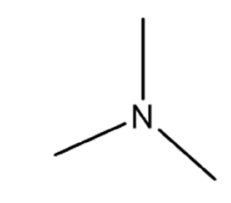

Trimethylamine

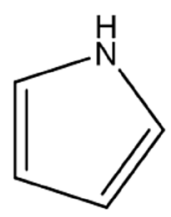

Pyrrole<smiles>NCCN</smiles>

Ethylenediamine<smiles>c1ccncc1</smiles>

Pyridine<smiles>Nc1ccccc1</smiles>

Aniline<smiles>c1cnccn1</smiles>

Pyrazine

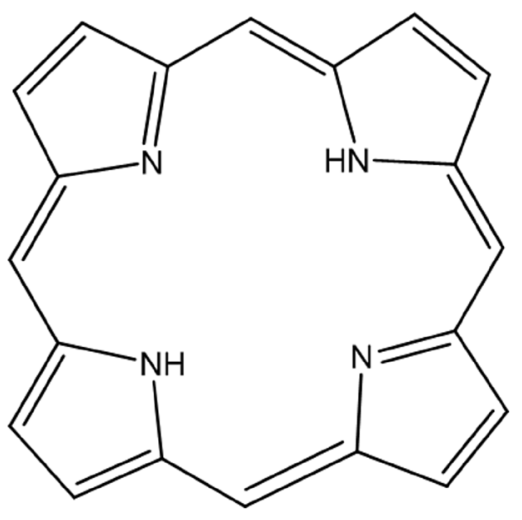

Porphin

Scheme 1 N-bases employed in the present study. The molecules differ on the hapticity, dispersion contributions and extension 
and $E_{\text {bulk }}$ the total energy of Au bulk per atom, and the factor $1 / 2$ accounts for the two surfaces in the slab unit-cell in an asymmetric configuration, relaxation taking place only on one the sides. The results, listed in Table 1, showed that (111) facet is most stable with a surface energy of $0.057 \mathrm{eV} /$ $\AA^{2}$. Our results are in reasonable agreement with the tests performed by Marzari [28]. We have also calculated the energy penalty to remove one atom from the lattice placing it as an adatom for (100), (110) and (111) facets and are 0.60, 1.00 and $1.20 \mathrm{eV}$. Therefore, the energy gain by adsorbing the ligand on the adatom with respect to the regular position needs to be larger than these thresholds to set a thermodynamic force for reconstruction.

Adsorption was only allowed on one of the sides of the Au slabs. We begin by calculating the adsorption energy for several different adsorption sites of the planar (100), (110), and (111) surfaces to ensure that the global minimum is found. The adsorption energies of the Ligand molecules were calculated with respect to the solution as follows:

$E_{\text {ads }}=\left(E_{S-\text { Ligand }}-E_{S}-E_{\text {Ligand }}^{\text {gas-phase }}\right)$

where $E_{S \text {-Ligand }}$ is the energy of the surface with the ligand molecule, $E_{S}$ is the energy of the simulation cell containing the surface only, and $E_{\text {Ligand }}^{\text {gas-phase }}$ is the energy of the molecule in the gas phase, calculated using the same conditions. Figure 1 shows the structure of the Ligand/Au systems after relaxation. It is revealed that the bridge and on-top adsorption sites for all molecules are the most stable ones with exothermic adsorption energies and that hollow sites are highly unfavorable. The adsorption energies for the adsorption of ligand molecules on different facets of $\mathrm{Au}$ surfaces reported in Table 1. The largest value for the adsorption energy $\left(E_{a d s}\right)$ per molecule is that for the $\mathrm{Au}(110)$ surface. Moreover, porphine has the strongest interaction with $\mathrm{Au}$ surfaces among all molecules. The average distances between $\mathrm{C}$ and $\mathrm{N}$ atoms of ligand and the first layer of $\mathrm{Au}$

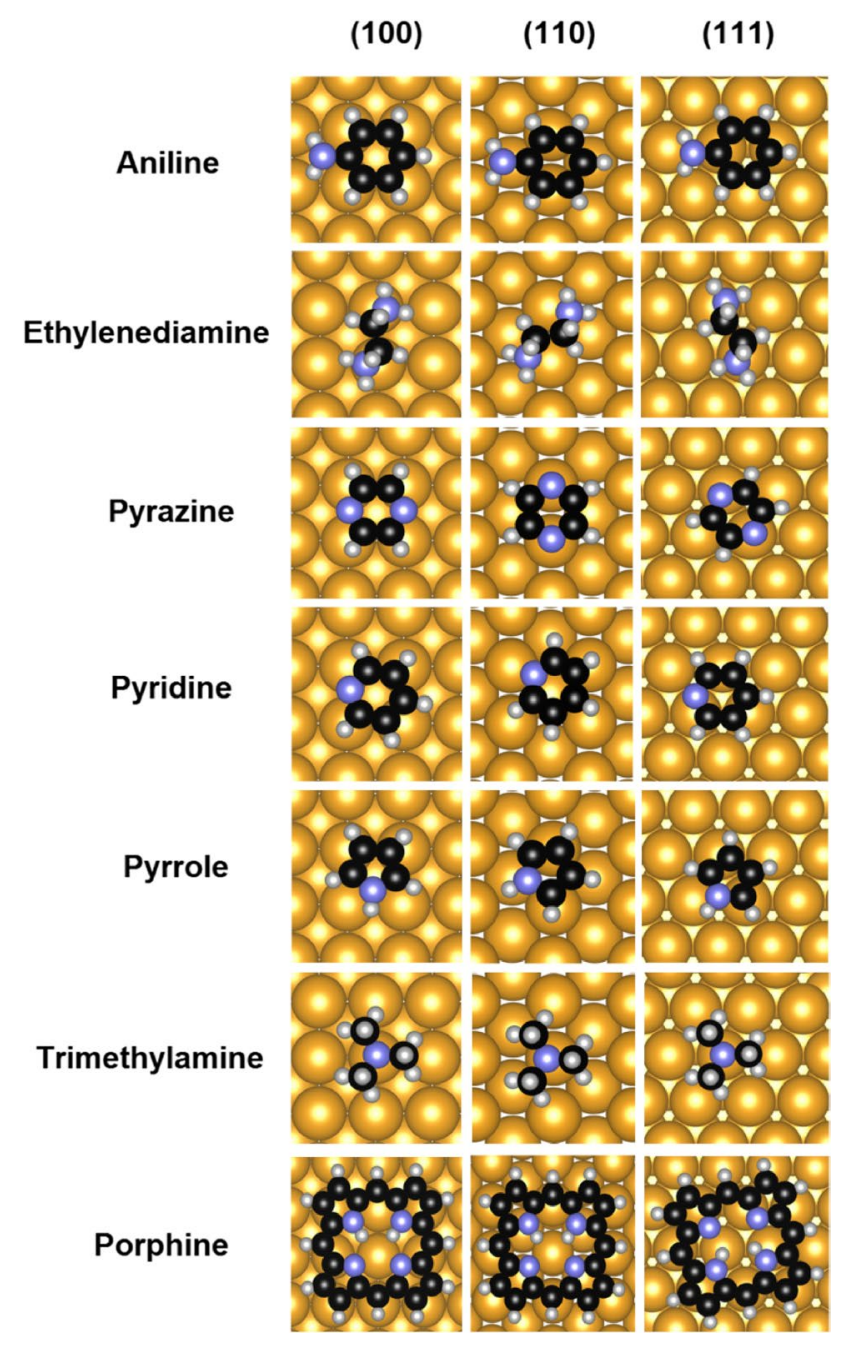

Fig. 1 Structures for the adsorption on the lowest energy surfaces. Color code: orange surface $\mathrm{Au}$ atoms, black $\mathrm{C}$ atoms, purple $\mathrm{N}$ atoms and gray $\mathrm{H}$ atoms
Table 1 Hapticity and adsorption energies, $\mathrm{E}_{\mathrm{ads}}$ in $\mathrm{eV}$, for the different ligands in this study on different $\mathrm{Au}$ surfaces

\begin{tabular}{|c|c|c|c|c|c|c|c|c|c|}
\hline \multirow[t]{2}{*}{ Ligand } & \multicolumn{3}{|c|}{ Hapticity } & \multicolumn{3}{|l|}{$E_{a d s}$} & \multicolumn{3}{|l|}{$\gamma^{\prime}$} \\
\hline & (111) & (100) & (110) & (111) & (100) & (110) & (111) & (100) & (110) \\
\hline None & & & & & & & 0.057 & 0.064 & 0.066 \\
\hline Aniline & 4 & 4 & 2 & -0.553 & -0.569 & -0.992 & 0.048 & 0.056 & 0.049 \\
\hline Ethylenediamine & 2 & 2 & 2 & -0.797 & -0.927 & -1.312 & 0.037 & 0.044 & 0.032 \\
\hline Pyrazine & 4 & 4 & 2 & -0.230 & -0.204 & -0.543 & 0.053 & 0.061 & 0.061 \\
\hline Pyridine & 3 & 4 & 2 & -0.289 & -0.262 & -0.301 & 0.052 & 0.060 & 0.057 \\
\hline Pyrrole & 3 & 4 & 2 & -0.470 & -0.454 & -0.807 & 0.049 & 0.057 & 0.052 \\
\hline Trimethylamine & 1 & 1 & 1 & -0.607 & -0.615 & -0.903 & 0.047 & 0.055 & 0.050 \\
\hline Porphine & 9 & 9 & 9 & -1.49 & -1.55 & -2.10 & 0.049 & 0.056 & 0.058 \\
\hline
\end{tabular}

Adsorption corrected surface energies, $\gamma^{\prime}$ in $\mathrm{eV} / \AA^{2}$, for the different molecules in this study on different $\mathrm{Au}$ surfaces 
slab are presented in Table 2. It can be seen that the smallest distance between ligands and Au surface is that for (110) facet which lead to the largest value of $\mathrm{E}_{\mathrm{ads}}$. The gas-phase adsorption energies are used in Table 1, when the relevant experiments may be done in the liquid phase. To probe the effects of solvation on adsorption energies of ligands on $\mathrm{Au}$ surface we applied recently developed a continuum solvation model into the VASP, a plane-wave based electronic structure code, VASP-MGCM (VASP-Multigrid Continuum Model) [29]. Water solvation of ethylene diamine is exothermic by $0.27 \mathrm{eV}$. Therefore, the binding energies reported for this ligand in Table 1 would be reduced to -0.6 for the (111) surface and about $-1.1 \mathrm{eV}$ for (110).

To further discern the interaction between ligands and $\mathrm{Au}$ surface charge density difference between pyrazine (weak interaction), aniline (moderate interaction) and ethylenediamine (strong interaction) ligands and different facets of $\mathrm{Au}$ slab has been calculated and illustrated in Fig. 2. It can be seen that electrons are accumulated under the vacuum region and the middle part between the ligands and the Au atoms. The accumulation of more electrons in the middle part creates the stronger interactions between the ligands and the $\mathrm{Au}$ surface. The pyrazine has a weak interaction on all Au facets leading to small influence on gold nanoparticle shape while the ethylenediamine has a big influence on the shape of nanoparticle due to strong interaction with Au surfaces. The interaction between aniline and (110) facet is strong unlike (100) and (111) facets hence it has a bigger influence on (110) facet than other ones. In order to analyze the effect of each ligand on gold nanoparticle shape the adsorption corrected surface energies are calculated.

To rationalize the strength of the bond at the interface we have looked at the trends behind adsorption. Particularly, the basicity of the ligand controls the adsorption. In particular, we have plotted the normalized (per free $\mathrm{N}$ atom) $\mathrm{E}_{\mathrm{ads}} / \mathrm{N}$ as a function of the HOMO position of the ligands, see Fig. 3. The strongest interactions always correspond to the (110) surface. For all surfaces, a linear dependence is found, showing the predominant role of the basicity of the ligand in the adsorption.

When the Au nanoparticle interacts with the surrounding environment, the surface energy is replaced by the adsorption corrected surface energies (interface tensions), $\gamma^{\prime}$, between $\mathrm{Au}$ and its environment which at $\mathrm{T}=0 \mathrm{~K}$ is given by

$\gamma^{\prime}=\gamma_{s}+\frac{f E_{a d s}}{A}$
Table 2 Average distance between $\mathrm{N}$ or $\mathrm{C}$ atoms of the ligands and the topmost $\mathrm{Au}$ surface in $\AA$

Fig. 2 Cross-sectional views of the charge density difference for pyrazine, aniline and ethylenediamine on different facets of $\mathrm{Au}$ surface for negative charge carriers $\left(a_{0}\right.$ : Bohr radius). Color code: like Fig. 1

\begin{tabular}{|c|c|c|c|c|c|c|}
\hline \multirow[t]{2}{*}{ Ligand } & \multicolumn{2}{|c|}{ (111) } & \multicolumn{2}{|c|}{$(100)$} & \multicolumn{2}{|c|}{ (110) } \\
\hline & $\mathrm{N}$ & $\mathrm{C}$ & $\mathrm{N}$ & $\mathrm{C}$ & $\mathrm{N}$ & $\mathrm{C}$ \\
\hline Aniline & 3.59 & 3.54 & 3.50 & 3.44 & 2.83 & 2.66 \\
\hline Ethylenediamine & 2.57 & 3.67 & 2.60 & 3.74 & 2.04 & 2.35 \\
\hline Pyrazine & 3.96 & 3.96 & 4.03 & 4.03 & 3.35 & 3.41 \\
\hline Pyridine & 3.81 & 3.82 & 3.84 & 3.87 & 2.32 & 3.06 \\
\hline Pyrrole & 3.42 & 3.42 & 3.41 & 3.42 & 2.72 & 2.62 \\
\hline Trimethylamine & 2.98 & 3.43 & 2.98 & 3.42 & 2.48 & 2.89 \\
\hline Porphine & 3.33 & 3.33 & 3.02 & 3.05 & 2.52 & 2.60 \\
\hline
\end{tabular}

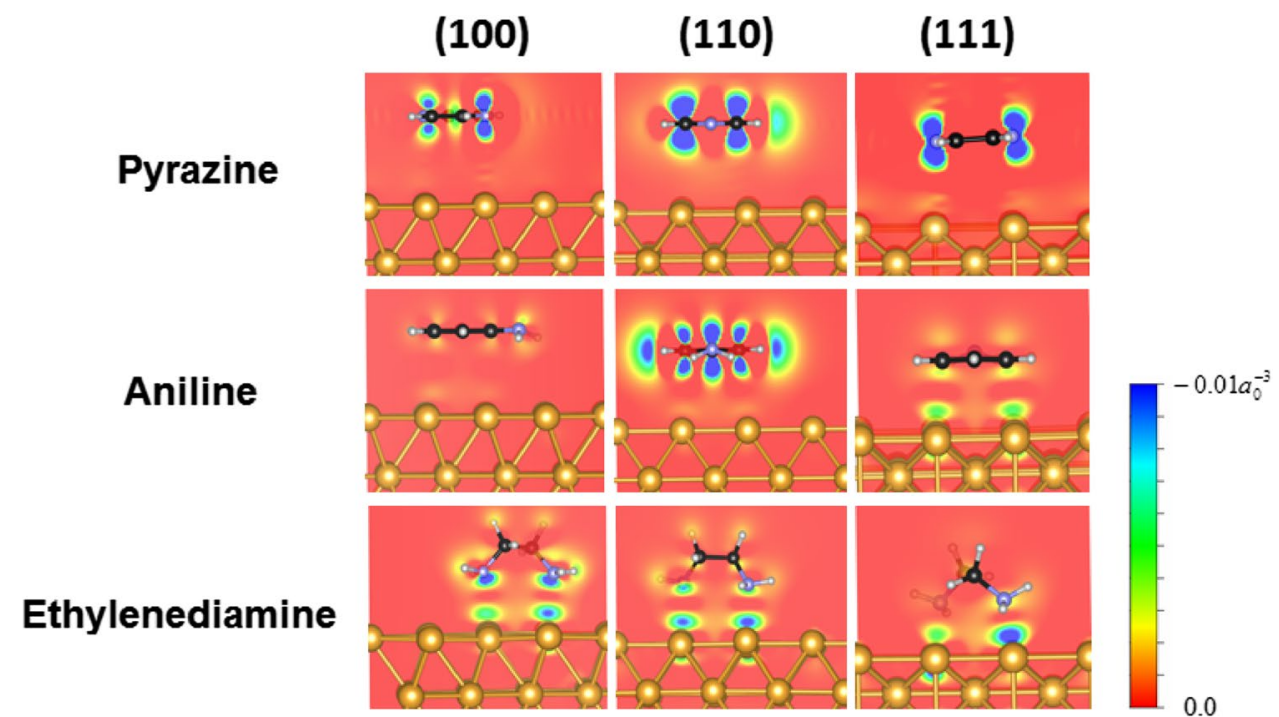




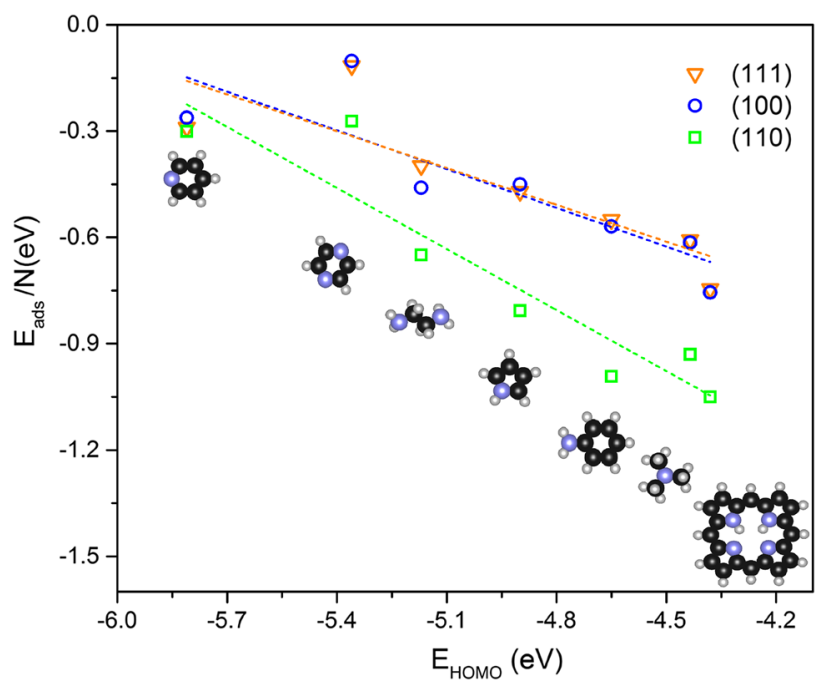

Fig. 3 Normalized (per free $\mathrm{N}$ atom) $\mathrm{E}_{\mathrm{ads}} / \mathrm{N}$ as a function of the HOMO position of the ligands

where $f$ is the area of each surface facet to the minimum area required for ligands adsorption on the Au surfaces $f=\frac{S_{\text {cell }}}{S_{\text {mol }}}$ assuming that the molecules lay flat. This is an approximation as at very high coverages the molecules could be adsorbed through the $\mathrm{N}$ atoms leaving the pi-systems interacting out of the surface; however this assumption simplifies the discussion and is relevant in a certain ligand concentration regime close to saturation. In our calculations we used $f=2$ for all ligand molecules adsorption except

ethylenediamine and porphine for which values of 3 and 1 were used, respectively. The adsorption corrected surface energies for all molecules and different Au facets are listed in Table 1. It can be seen that the (111) facet is still the most stable surface after adsorbing molecules and other $(h k l)$ planes have much higher surface energies. The equilibrium morphology of the nanoparticles was obtained through the Wulff construction method [30] which depends on the relative $\gamma^{\prime}$ from Table 1. Figure 4 illustrates the Wulff structures for bare Au nanoparticle and ligand-covered Au system. The crystal morphology model was created with VESTA (Visualization for Electronic and Structural Analysis) version 3.4.0 package [31]. We also calculated total volume and fraction of each of the surfaces as a function of ligand for a nanoparticle with a radius of $5 \mathrm{~nm}$ (Table 3 ). The total volume for bare Au nanoparticle is $73.42 \mathrm{~nm}^{3}$ where the dominant facet is (111) surface with the fraction surface value of 0.65 , in good agreement with previous works $[4,13,28,32]$. From Fig. 4 and Table 3, it can be concluded that for all molecules adsorption except aniline and ethylenediamine the dominant facet is still (111) while for two latest ligands, nanoparticle surface is dominated by (110) facet. In addition, the pyrazine, pyridine and porphine molecules have a very small influence on $\mathrm{Au}$ nanoparticle morphology after adsorption while aniline, ethylenediamine, pyrrole and trimethylamine completely change the morphology of $\mathrm{Au}$ nanoparticle. For the latest molecules the fraction of (110) facet increases in comparison with bare Au nanoparticle while regions of (111) facet decrease. Regions for (100) facet is almost same for the pyrazine, pyridine and porphine

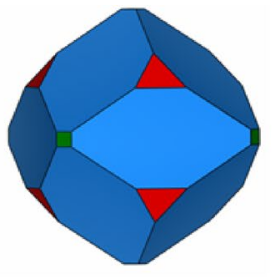

Ethylenediamine

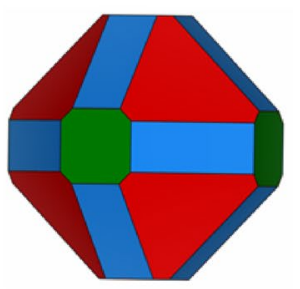

Trimethylamine

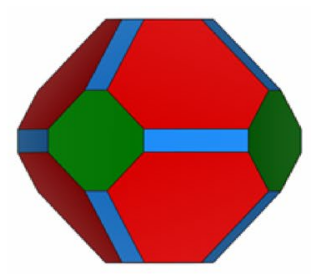

Pyrazine

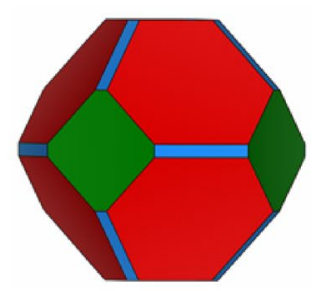

Porphine

Fig. 4 Wulff constructions for gold decorated nanoparticles as a function of the adsorbate: red planes are (111), green planes are (100) and blue planes are (110) facets 
Table 3 Total volume, fraction of each of the surfaces as a function of ligand for a nanoparticle with a diameter of $5 \mathrm{~nm}$

\begin{tabular}{lllll}
\hline Ligand & Volume $\left(\mathrm{nm}^{3}\right)$ & $(111)$ & $(100)$ & $(110)$ \\
\hline Bare $\mathrm{Au}$ & 73.42 & 0.65 & 0.25 & 0.10 \\
Aniline & 72.59 & 0.31 & 0.12 & 0.57 \\
Ethylenediamine & 57.20 & 0.05 & 0.01 & 0.94 \\
Pyrazine & 73.42 & 0.63 & 0.22 & 0.15 \\
Pyridine & 73.42 & 0.52 & 0.19 & 0.29 \\
Pyrrole & 69.96 & 0.43 & 0.15 & 0.42 \\
Trimethylamine & 69.96 & 0.46 & 0.16 & 0.38 \\
Porphine & 74.90 & 0.70 & 0.23 & 0.07 \\
\hline
\end{tabular}

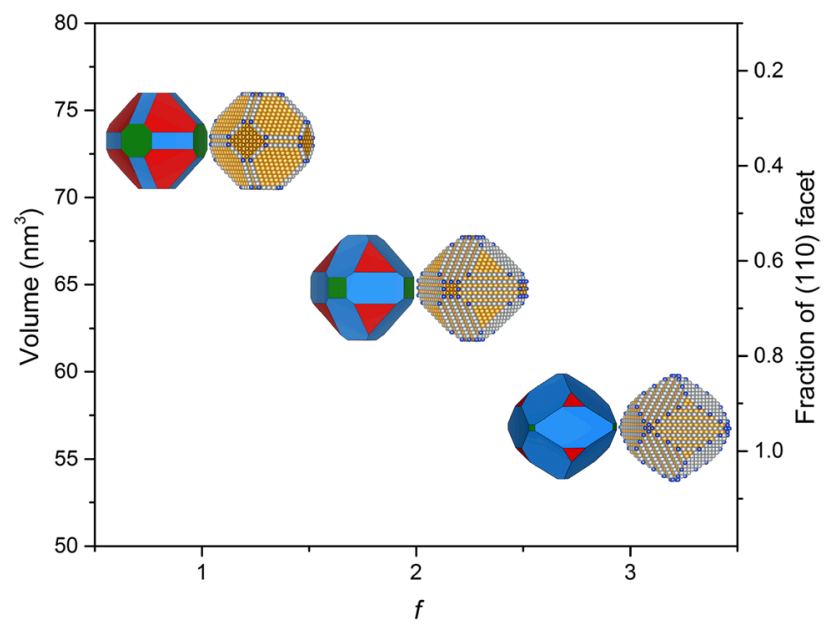

Fig. 5 Wulff and atomistic Wulff constructions for ethylenediaminecovered Au system at different coverages. Color code as in Fig. 4

molecules after adsorption while it is decreased in other ones.

To show the effect of molecular coverage on the Au nanoparticle shape, both the continuous and the atomistic Wulff structure of ethylenediamine-covered Au system at different coverages is plotted versus nanoparticle volume (and fraction of (110) facet) in Fig. 5. As it can be seen by increasing ethylenediamine coverage the fraction of (110) facet increases while nanoparticle volume and regions of (111) and (100) facets decrease. The total volume for ethylenediamine-covered Au nanoparticle is 73.42, 65.37 and 57.20 $\mathrm{nm}^{3}$ for $f=1,2$ and 3 , respectively. The related values of the fraction of (110) facet is 31,70 and $94 \%$ for same coverages. Similarly from the atomistic Wulff construction the number of low coordinated atoms can be retrieved. For the lowest coverage the fraction of low coordinated $(\mathrm{NC}=3)$ atoms is $1.2 \%$ and $\mathrm{NC}=5$ is about $5 \%$. Instead at higher ligand content these two fractions raise to 1.7 and $13 \%$. Finally, for the highest ethylene diamine content the fraction of these atoms are 2.1 and $15 \%$, respectively.
In summary, the adsorption of N-bases can modify significantly the shape of nanoparticles increasing facets that are not expressed in clean gold nanoparticles. This will modify the catalytic properties in two directions as electron modifiers and for reactions with structure sensitivity as a different facet is present is the most common one in ethylenediamine.

\section{Conclusions}

We have constructed and characterized gold nanoparticle morphology in thermodynamic equilibrium, including interactions with 7 different ligand molecules (aniline, ethylenediamine, pyrazine, pyridine, pyrrole, trimethylamine and porphine) using the Wulff construction. We first studied low-index Au surfaces [(100), (110) and (111)], both clean and with ligand molecules adsorbed on them. We found that the bridge and on-top adsorption sites for all molecules are the most stable ones with exothermic adsorption energies. Adsorption is found to correlate with the number of $\mathrm{N}$ atoms in contact with the surface and the HOMO (donor) ability of the ligand.

The adsorption of pyrazine, pyridine and porphine molecules does not change the Au nanoparticle morphology while the adsorption of aniline, ethylene diamine, pyrrole and trimethylamine increases regions of (110) facet and decreases regions of (111) facet, leading a major change in $\mathrm{Au}$ nanoparticle morphology. Particularly for ethylene diamine the change of the shape with concentration illustrates the important role of small ligands in the final shape of nanoparticles. As both the nature of the ligands and the induced shape changes might affect adsorption and reaction on nanoparticles a full design of the interface will be required to fully benefit from the synergetic effects at the interface.

Acknowledgements This work was funded by the MINECO (Grant Number CTQ2015-68770-R) and Marie Curie-COFUND (Grant Number 291787-ICIQ-IPMP, M.S.). M. Domingo thanks Fundació la Caixa for a Summer Fellowship at ICIQ. We would like to thank BSC-RES for generous computational resources.

\section{References}

1. Daniel M-C, Astruc D (2004) Gold nanoparticles: assembly, supramolecular chemistry, quantum-size-related properties, and applications toward biology, catalysis, and nanotechnology. Chem Rev 104(1):293-346

2. Hvolbæk B et al (2007) Catalytic activity of Au nanoparticles. Nano Today 2(4):14-18

3. Falsig $\mathrm{H}$ et al (2008) Trends in the catalytic $\mathrm{CO}$ oxidation activity of nanoparticles. Angew Chem Int Ed 47(26):4835-4839

4. Barmparis GD, Remediakis IN (2012) Dependence on CO adsorption of the shapes of multifaceted gold nanoparticles: a density functional theory. Phys Rev B 86(8):085457 
5. Siler CGF et al (2014) Switching selectivity in Oxidation reactions on gold: the mechanism of $\mathrm{C}-\mathrm{C}$ vs $\mathrm{C}-\mathrm{H}$ bond activation in the acetate intermediate on $\mathrm{Au}(111)$. ACS Catal 4(9):3281-3288

6. Cremer T et al (2014) Tuning the stability of surface intermediates using adsorbed oxygen: acetate on $\mathrm{Au}(111)$. J Phys Chem Lett 5(7):1126-1130

7. Kosuda KM et al (2012) Oxygen-mediated coupling of alcohols over nanoporous gold catalysts at ambient pressures. Angew Chem Int Ed 51(7):1698-1701

8. Stowers KJ, Madix RJ, Friend CM (2013) From model studies on $\mathrm{Au}(111)$ to working conditions with unsupported nanoporous gold catalysts: oxygen-assisted coupling reactions. J Catal 308(Supplement C): 131-141

9. Stephen A, Hashmi K (2004) Homogeneous catalysis by gold. Gold Bull 37(1):51-65

10. Heinz $\mathrm{H}$ et al (2017) Nanoparticle decoration with surfactants: molecular interactions, assembly, and applications. Surf Sci Rep 72(1): $1-58$

11. Barmparis GD, Honkala K, Remediakis IN (2013) Thiolate adsorption on $\mathrm{Au}(\mathrm{hkl})$ and equilibrium shape of large thiolatecovered gold nanoparticles. J Chem Phys 138(6):064702

12. Barmparis GD et al (2015) Nanoparticle shapes by using Wulff constructions and first-principles calculations. Beilstein J Nanotechnol 6:361-368

13. Almora-Barrios $\mathrm{N}$ et al (2014) Theoretical description of the role of halides, silver, and surfactants on the structure of gold nanorods. Nano Lett 14(2):871-875

14. Gómez-Graña S et al (2013) Au@ Ag nanoparticles: halides stabilize $\{100\}$ facets. J Phys Chem Lett 4(13):2209-2216

15. Li Q et al (2017) Shape control in concave metal nanoparticles by etching. Nanoscale 9(35):13089-13094

16. Chen $G$ et al (2016) Interfacial electronic effects control the reaction selectivity of platinum catalysts. Nat Mater 15(5):564-569

17. Almora-Barrios $\mathrm{N}$ et al (2017) Concerted chemoselective hydrogenation of acrolein on secondary phosphine oxide decorated gold nanoparticles. ACS Catal 7(6):3949-3954

18. Fiorio JL, López N, Rossi LM (2017) Gold-ligand-catalyzed selective hydrogenation of alkynes into cis-alkenes via $\mathrm{H} 2$ heterolytic activation by frustrated Lewis Pairs. ACS Catal 7(4):2973-2980

19. Arndt S, Rudolph M, Hashmi ASK (2017) Gold-based frustrated Lewis acid/base pairs (FLPs). Gold Bull 50(3):267-282

20. Kresse G, Furthmüller J (1996) Efficient iterative schemes for ab initio total-energy calculations using a plane-wave basis set. Phys Rev B 54(16):11169-11186

21. Kresse G, Furthmüller J (1996) Efficiency of ab-initio total energy calculations for metals and semiconductors using a plane-wave basis set. Comput Mater Sci 6(1):15-50

22. Perdew JP, Burke K, Ernzerhof M (1996) Generalized gradient approximation made simple. Phys Rev Lett 77(18):3865-3868

23. Kresse G, Joubert D (1999) From ultrasoft pseudopotentials to the projector augmented-wave method. Phys Rev B 59:1758

24. Grimme S (2006) Semiempirical GGA-type density functional constructed with a long-range dispersion correction. J Comput Chem 27(15):1787-1799

25. Bučko T et al (2010) Improved description of the structure of molecular and layered crystals: ab initio DFT calculations with van der Waals corrections. J Phys Chem A 114(43):11814-11824

26. Almora-Barrios $\mathrm{N}$ et al (2014) Costless derivation of dispersion coefficients for metal surfaces. J Chem Theory Comput 10(11):5002-5009

27. Álvarez-Moreno M et al (2015) Managing the computational chemistry big data problem: the ioChem-BD platform. J Chem Inf Model 55(1):95-103

28. Singh-Miller NE, Marzari N (2009) Surface energies, work functions, and surface relaxations of low-index metallic surfaces from first principles. Phys Rev B 80(23):235407

29. Garcia-Ratés M, López N (2016) Multigrid-based methodology for implicit solvation models in periodic DFT. J Chem Theory Comput 12(3):1331-1341

30. Wulff G (1901) Zeitschrift fur Krishtallographie 34:449-530

31. Appl J (2011) VESTA 3 for three-dimensional visualization of crystal, volumetric and morphology data. J Appl Crystallogr 44:1272-1276

32. Shi H, Stampfl C (2008) Shape and surface structure of gold nanoparticles under oxidizing conditions. Phys Rev B 77(9):094127 